\title{
Proposal of multiple-slot silica high-mesa waveguide for infrared absorption
}

\author{
Jiao Chen ${ }^{\mathrm{a})}$, Hirohito Hokazono, Miki Tsujino, \\ Daisuke Nakashima, and Kiichi Hamamoto \\ Interdisciplinary Graduate School of Engineering Science, Kyushu University, \\ 6-1 Kasugakouen, Kasuga, Fukuoka 816-8580, Japan \\ a)chenjiao0@asem.kyushu-u.ac.jp
}

Abstract: We propose a multiple-slot silica high-mesa waveguide for infrared sensing considering the possibility of realizing a higher portion of an optical field out of the waveguide (which is defined as “ $\Gamma_{\text {air }}$ "). Low $\Gamma_{\text {air }}$ leads to less light power being used for sensing, which limits sensing capabilities. The simulated results showed a high $\Gamma_{\text {air }}$ of $20.3 \%$ for a quadruple structure under $\lambda=1550 \mathrm{~nm}$. A scattering loss of $0.06 \mathrm{~dB} / \mathrm{cm}$ was predicted theoretically as well.

Keywords: infrared sensing, silica high-mesa

Classification: Optoelectronics, Lasers and quantum electronics, Ultrafast optics, Silicon photonics, Planar lightwave circuits

\section{References}

[1] H. Dahnke, D. Kleine, P. Hering and M. Mürtz: Appl. Phys. B 72 (2001) 971.

[2] A. Yalcin, K. C. Popat, J. C. Aldridge, T. A. Desai, J. Hryniewicz, N. Chbouki, B. E. Little, O. King, V. Van, S. Chu, D. Grill, M. A. Washburn, M. S. Unlu and B. B. Goldberg: IEEE J. Sel. Topics Quantum Electron. 12 [1] (2006) 148.

[3] B. J. Luff, R. D. Harris, J. S. Wilkinson, R. Wilson and D. J. Schiffrin: Opt. Lett. 21 [8] (1996) 618.

[4] C. Y. Chao, W. Fung and L. J. Guo: IEEE J. Sel. Topics Quantum Electron. 12 [1] (2006) 134.

[5] N. Marczin, S. A. Kharitonov, M. Yacoub and P. J. Barnes: Disease Markers in Exhaled Breath (Lung Biology in Health and Disease) (Marcel Dekker, Inc., New York, 2003) 3.

[6] T. H. Risby and S. F. Solga: Appl. Phys. B 85 [2-3] (2006) 421.

[7] G. von. Basum, H. Dahnke, D. Halmer, P. Hering and M. Murtz: J. Appl. Physiol. 95 (2003) 2583.

[8] T. Tsuchizawa, K. Yamada, H. Fukuda, T. Watanabe, J. Takahashi, M. Takahashi, T. Shoji, E. Tamechika, S. Itabashi and H. Morita: IEEE J. Sel. Topics Quantum Electron. 11 (2005) 232.

[9] S. Yano, K. Kameyama and K. Hamamoto: Tech. Dig. IPNRA (2007) IWA7.

[10] C. Shi, C. Lu, C. Gu, L. Tian, R. Newhouse, S. Chen and J. Z. Zhang: Appl. Phys. Lett. 93 [15] (2008) 153101.

[11] A. Wilk, F. Seichter, S. Kim, E. Tutuncu, B. Mizaikoff, J. A. Vogt, U. Wachter and P. Radermacher: Anal. Bioanal. Chem. 402 [1] (2012) 397. 
[12] J. T. Robinson, L. Chen and M. Lipson: Opt. Express 16 [6] (2008) 4296.

[13] W. C. Lai, S. Chakravarty, X. Wang, C. Lin and R. T. Chen: Opt. Lett. 36 [6] (2011) 984.

[14] S. Sato, T. Katagiri and Y. Matsuura: J. Opt. Soc. Am. B 29 [11] (2012) 3006.

[15] A. Kriesch, S. P. Burgos, D. Ploss, H. Pfeifer, H. A. Atwater and U. Peschel: Nano Lett. 13 [9] (2013) 4539.

[16] A. Intekhab, Y. Matsunaga, S. Hirofuji, T. Mitomi, T. Murayama, Y. Kokaze, H. Wado, Y. Takeuchi and K. Hamamoto: Tech. Dig. MOC 2009, F3.

[17] I. Alam and K. Hamamoto: Jpn. J. Appl. Phys. 49 [12] (2010) 122503-1.

[18] J. Chen, H. Hokazono, D. Nakashima, Y. Hashizume, M. Itoh and K. Hamamoto: Tech. Dig. CLEL-PR\&OECC/PS 2013, ThL1-1.

[19] A. R. Davoyan, V. V. Popov and S. A. Nikitov: Phys. Rev. Lett. 108 (2004) 127401.

[20] R. W. Ricci, M. Ditzler and L. P. Nestor: J. Chem. Educ. 71 [11] (1994) 983.

\section{Introduction}

The demand for a compact health-check system that can perform routine health-care checks has increased recently. Infrared absorption [1] based breath-content analysis using an optical integrated circuit has been studied widely for health monitoring $[2,3,4]$ given the presence of various diseasemarkers $[1,5,6]$ in breath-contents and the ppm-order sensing ability of infrared absorption. Additionally, the use of optical integrated circuits [7] is a promising solution for realizing the meter-long optical path length of regular gas cell $[1,6,8,9]$ within a compact area considering the capability of realizing small radius of curvature. One of the critical issues with infrared sensing is the portion of light profiles outside of the waveguide $\left(\Gamma_{\text {air }}\right)$, which can be used for sensing. Low $\Gamma_{\text {air }}$ leads to less light power being used for sensing, thus limiting the sensing capability. In this paper, we propose a multiple-slot silica high-mesa waveguide in the light of its possibility of realizing higher $\Gamma_{\text {air }}$. The reasons underlying our proposal are as follows. Firstly, so far, several types of waveguide structures have been proposed for infrared sensing, such as the hollow $[10,11]$ and the slot $[12,13]$ structures. Compared with the hollow structure, the slot structure is attractive for realizing a smaller radius of curvature [14, 15], which is helpful for realizing high-density integration. Secondly, among several types of slot waveguides, the slot waveguide using a high-mesa structure [16] is useful because it offers a higher portion of an optical field's profile inside the slot region than the others [17]. Thirdly, according to our previous research, a low propagation loss of $0.02 \mathrm{~dB} / \mathrm{cm}$ (@ $\lambda=1550 \mathrm{~nm}, \mathrm{w}=2.3 \mu \mathrm{m})$ [18] has been achieved using a silica-based high-mesa waveguide. However, the $\Gamma_{\text {air }}$ of the silica high-mesa waveguide was only 2.2\%(@ $\lambda=1550 \mathrm{~nm}, \mathrm{w}=$ $2.3 \mu \mathrm{m})$. This is because the small difference in the refractive indices of silica high-mesa and air leads to a low electric field discontinuity at the high-mesa-air interface, and thus a relatively low $\Gamma_{\text {air }}$ for single high-mesa structures. Therefore, the proposed multiple-slot silica high-mesa wave- 
guide is expected to be able to solve this problem. In the meantime, low propagation loss is expected as well because propagation loss consumes light power, thus restricting the sensing capability. The simulated results showed a high $\Gamma_{\text {air }}$ of $20.3 \%$ for a quadruple structure under a wavelength of $1550 \mathrm{~nm}$. In addition, a low scattering loss of $0.06 \mathrm{~dB} / \mathrm{cm}$ was confirmed theoretically.

\section{Concept of multiple slot high-mesa waveguide}

Fig. 1 shows the waveguide cross-sections of different waveguide structures, namely, (a) single silica high-mesa waveguide, (b) double high-mesa waveguide, (c) triple high-mesa waveguide, and (d) quadruple high-mesa waveguide. The geometry parameters of all structures were set as $5 \mu \mathrm{m}$, $3.5 \mu \mathrm{m}$, and $5 \mu \mathrm{m}$ for the cover cladding layer, core, and under cladding layer, respectively [18]. As shown in Fig. 1 (a), for the single structure, most of the optical power is confined in the solid material, and only a few optcial profiles can be detected outside of the solid material. This leaves a low amount of light power for infrared sensing. In contrast, compared with the single structure, the slot structures shown in Figs. 1 (b), (c), and (d) show a great number of optical power profiles out of the waveguide, especially in the slot regions. This is because multiple-slot high-mesa structures comprise several parallel conventional single high-mesas adjacent to each other, and the slot width between mesas is only of the order of nanometers. Thus, the near-field effect enhances the optical power portion inside the slot region [19], and higher $\Gamma_{\text {air }}$ can be expected from the multiple structure. The optical fields were simulated using the finite element method (FEM). The TE mode was used for obtaining all simulation results in this study.
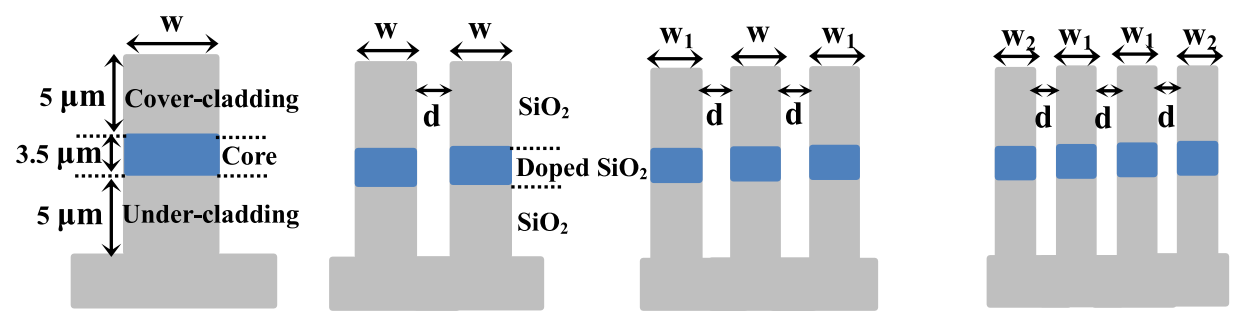

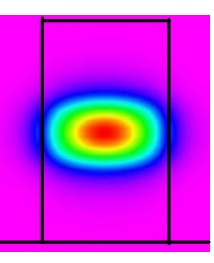

$\mathrm{w}=2.3 \mu \mathrm{m}$

(a)

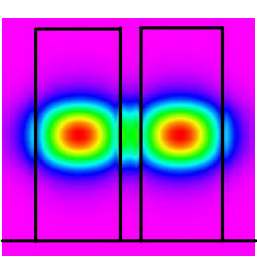

$\mathrm{W}=1.4 \mu \mathrm{m}, \mathrm{d}=400 \mathrm{~nm}$

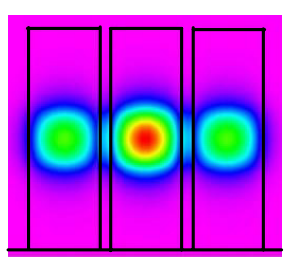

$\mathrm{w}_{1}=1.9 \mu \mathrm{m}, \mathrm{w}_{1}=1.9 \mu \mathrm{m}$ $\mathrm{d}=350 \mathrm{~nm}$

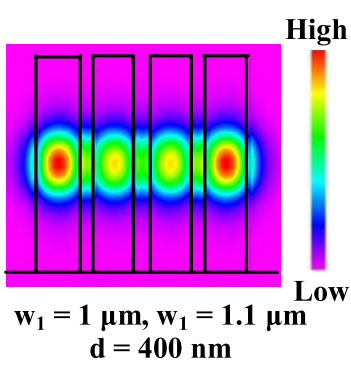

(d)

Fig. 1. Schematics of waveguide cross-sections and simulated optical profiles. The parameters are shown on set of the figures. (a) Single silica highmesa, (b) double high-mesa, (c) triple highmesa, (d) quadruple high-mesa. 


\section{Results and discussion}

Fig. 2 shows the $\Gamma_{\text {air }}$ as a function of the numbers of mesa (the maximum of $\Gamma_{\text {air }}$ for each number of mesas is shown). As shown in Fig. 2, the $\Gamma_{\text {air }}$ of the multiple-slot high-mesa is higher than that of the single high-mesa, as we expected, and the highest $\Gamma_{\text {air }}$ appears with the quadruple high-mesa structure. In addition, Fig. 2 shows that the $\Gamma_{\text {air }}$ of an even number of mesas is higher than that of an odd number of mesas. This is further confirmed in Fig. 1. Compared with the double and the quadruple structures, there are considerably more power profiles inside the middle mesa than in the slot regions in the triple structure, as shown in Fig, 1 (c), which results in a low $\Gamma_{\text {air. }}$. For a multiple-slot waveguide, internal waveguides (e.g., w for triple structures, $\mathrm{w}_{1}$ for quadruple structures) and slot regions can be considered as an equivalent inter-slot sandwiched between the two exteral waveguides. Thus, altering the numbers of internal wavegudies is equal to changing the effective index of this equivalent interslot. Owing to the symmetry of the propagation mode, the effective indices of this equivalent inter-slot of an odd number of mesas are higher than those of an even number of mesas. Therefore, the effective indices of an even number of mesas are lower than those of an odd number of mesas, which can be confirmed from Fig. 3 . This leads to the lower $\Gamma_{\text {air }}$ when using

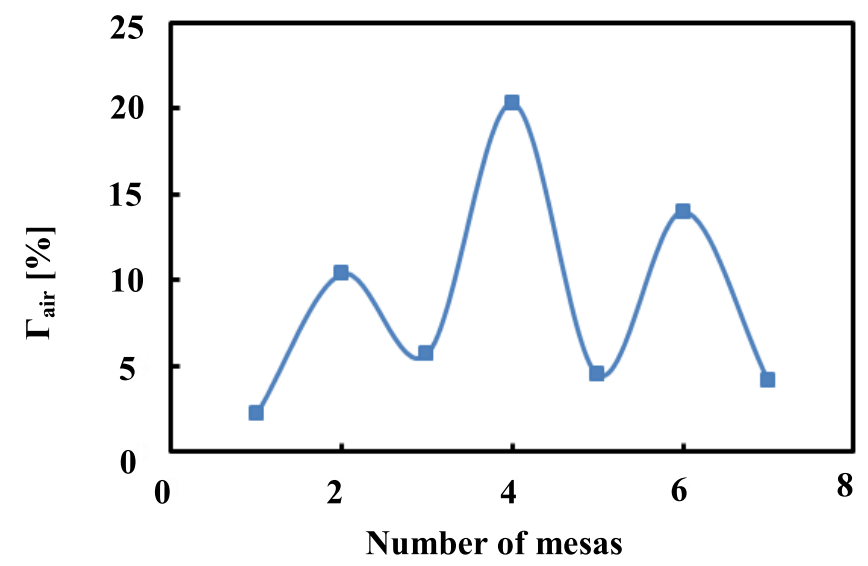

Fig. 2. $\Gamma_{\text {air }}$ as a function of number of mesas. $\lambda=1550 \mathrm{~nm}$ and the TE mode are used.

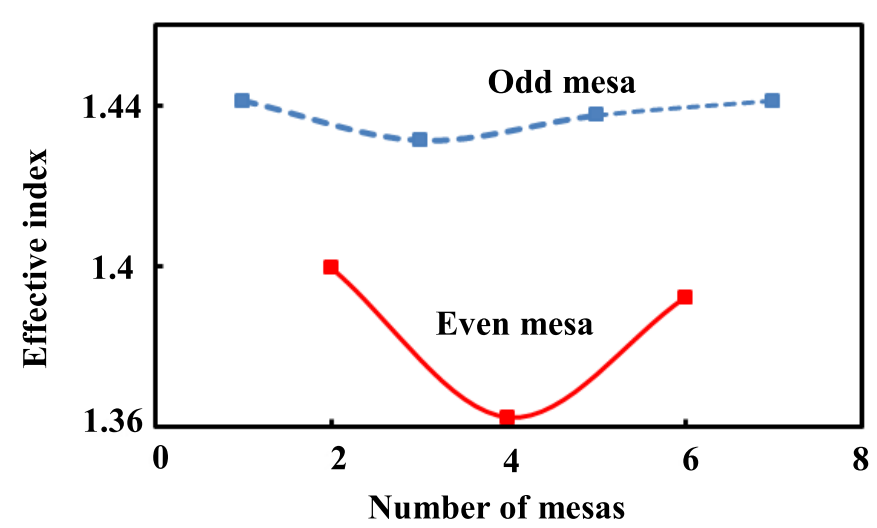

Fig. 3. Effective indices as a function of number of mesas for conventional and multiple-slot high-mesa waveguides. The geometry parameters are the same as those of the maximum $\Gamma_{\text {air }}$ for each structrue. 
an odd number of mesas.

Considering the analysis mentioned above, we propose a quadruple struture given its possibility of achieving the highest $\Gamma_{\text {air }}$. Given tha the optical field profile depends on the waveguide geometry of the waveguide width $\mathrm{w}$ and the slot widht $\mathrm{d}, \Gamma_{\text {air }}$ should be estimated depending on these parameters. Fig. 4 shows a theoretical estimation of $\Gamma_{\text {air }}$ as a function of the slot width (the maximum $\Gamma_{\text {air }}$ for each slot width is shown). From Fig. 4, the highest $\Gamma_{\text {air }}$ appears when the slot width $d=400 \mathrm{~nm}$. The region of $\mathrm{d}=$ $400 \mathrm{~nm} \pm 20 \%$ is marked in Fig. 4 as well. This region is a reasonable tolerance range for a slot width in actual fabrication. Compared with the peak, the difference in the maximum and minimum $\Gamma_{\text {air }}$ in this region is less than $2 \%$. This could be very helpful for facilitating the etching procedure. For the slot width of $\mathrm{d}=400 \mathrm{~nm}$, the results of altering $\mathrm{w}_{1}(0.8 \mu \mathrm{m} \sim$ $1.3 \mu \mathrm{m})$ and $\mathrm{w}_{2}(1 \mu \mathrm{m} \sim 1.4 \mu \mathrm{m})$ are summarized in Table I. The maximum $\Gamma_{\text {air }}$ reaches $20.3 \%$, which is almost ten times the value of the single structure for $\mathrm{w}_{1}=1 \mu \mathrm{m}$ and $\mathrm{w}_{2}=1.1 \mu \mathrm{m}$. There are no propagation modes when $\mathrm{w}_{1}<=1 \mu \mathrm{m}$ and $\mathrm{w}_{2}=1 \mu \mathrm{m}$.

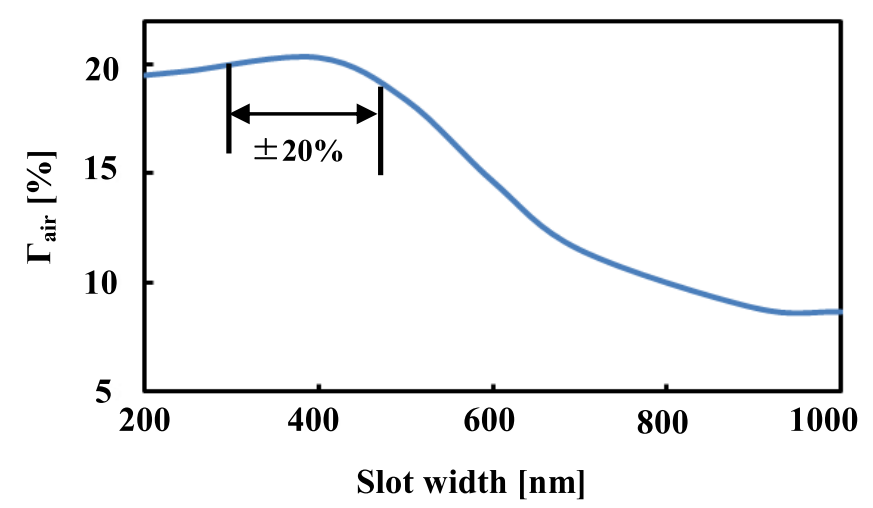

Fig. 4. $\Gamma_{\text {air }}$ as a funtion of slot width for quadruple structure. We use the TE mode.

Table I. $\Gamma_{\text {air }}$ of quadruple structure high-mesa. (Slot width $\mathrm{d}=400 \mathrm{~nm}, \lambda=1550 \mathrm{~nm}, \mathrm{TE}$ mode)

\begin{tabular}{|c|c|c|c|c|c|c|}
\hline \multirow{4}{*}{} & \multicolumn{5}{|c|}{$\mathrm{W} 2(\mu \mathrm{m})$} \\
\cline { 2 - 7 } & 0.8 & 1 & 1.1 & 1.2 & 1.3 & 1.4 \\
\cline { 2 - 7 } w1 & 0.9 & & $17.2 \%$ & $13.7 \%$ & $11.1 \%$ & $9.2 \%$ \\
\cline { 2 - 7 }$(\mu \mathrm{m})$ & 1 & & $18.1 \%$ & $14.1 \%$ & $11.3 \%$ & $8.9 \%$ \\
\cline { 2 - 7 } & 1.1 & $19.1 \%$ & $19.2 \%$ & $16.1 \%$ & $12.2 \%$ & $9.7 \%$ \\
\cline { 2 - 7 } & 1.2 & $15.7 \%$ & $15.9 \%$ & $15.8 \%$ & $11.4 \%$ & $13.3 \%$ \\
\cline { 2 - 7 } & 1.3 & $13.0 \%$ & $13.1 \%$ & $13.3 \%$ & $13.3 \%$ & $10.9 \%$ \\
\hline
\end{tabular}

In addition, we theoretically estimated the scattering loss of the quadruple structure waveguide using the beam propagation method (BPM) with the sidewall roughness was set to $10 \mathrm{~nm}$, which is the sidewall roughness value realized using the single structure [18]. A scattering loss of $0.06 \mathrm{~dB} / \mathrm{cm}$ is estimated for the quadruple structure. To identify the better alternative between the single high-mesa structure and the quadruple 
structure based on the above results, we consider the following criterion for evaluating waveguide performance in infrared sensing.

As mentioned above, the sensitivity of infrared absorption is influenced by both the propagation loss and the $\Gamma_{\text {air }}$. To compare the proposed quadruple structure with the single structure, we presented a new criterion that takes into account both the propagation loss and the $\Gamma_{\text {air }}$ for waveguide evaluation. According to the definition of propagation loss and the Lambert-Beer law [20], the intensity of incident light and transmitted light can be written as follows:

$$
I=I_{0} e^{-\sigma n L \Gamma_{a i r}} e^{-\alpha L} .
$$

Here, $I_{0}$ and $I$ denote the intensities of the incident light and the transmitted light, respectively, $\sigma$ denotes the cross section of light absorption by a single particle, $n$ denotes the density (number per unit volume) of the absorption particles, $L$ denotes the waveguide length, $\alpha$ denotes the propagation loss, and $\Gamma_{\text {air }}$ denotes the portion of optical field profiles outside the solid material. $e^{-\sigma n L \Gamma_{a i r}}$ and $e^{-\alpha L}$ correspond to the infrared absorption attenuation and propagation loss attenuation, respectively. We define a new variable $A$ as follows:

$$
A=\frac{e^{-\alpha L}}{e^{-\sigma n L \Gamma_{a i r}}}=e^{\alpha L\left(\sigma n \frac{\Gamma_{a i r}}{\alpha}-1\right)} .
$$

$A$ can be used for evaluating the waveguides' performance; a higher $A$ favors higher infrared absorption sensitivity. $\alpha L$, on the right side of equation (2), denotes the propagation loss of the entire waveguide and can be controlled by adjusting the waveguide length; therefore, it can be considered as a constant. For a certain concentration gas, $\sigma$ and $n$ can also be considered as constants. Then, $A$ is decided by $B=\Gamma_{\text {air }} / \alpha$. The higher the $B$ value, the higher is the value of $A$. The waveguide can be evaluated by substituting the parameters into $B$. For instance, for the single silica high-mesa waveguide, $\alpha=0.02 \mathrm{~dB} / \mathrm{cm}$ and $\Gamma_{a i r}=2.2 \%$, thus leading to $B_{\text {single }}=1.1$. For the proposed quadruple structure, $\alpha=0.06 \mathrm{~dB} / \mathrm{cm}$ and $\Gamma_{\text {air }}=20.3 \%$, thus leading to $B_{\text {quadruple }}=3.4$. From this view-point, the proposed quadruple structure is better for infrared absorption. Furthermore, the sidewall roughness of the silica structure was $10 \mathrm{~nm}$ [18], which is considerably high compared with that of the SOI structure $(1 \mathrm{~nm})$ [16]. Therefore, we believe that for this multiple-slot silica high-mesa structure, a further reduction of the propagation loss is possible through improvement of sidewall roughness. For a sidewall roughness of $1 \mathrm{~nm}$, the propagation loss of the proposed quadruple silica structure has been confirmed to be as low as $0.005 \mathrm{~dB} / \mathrm{cm}$ through BPM simulation. Concurrently, an extremely high $B_{\text {quadruple }}$ of 40.6 can be achieved.

\section{Conclusions}

We proposed a multiple-slot silica high-mesa waveguide and found that the $\Gamma_{\text {air }}$ when using an even number of mesas is always higher than that when using an odd number of mesas. Theoretically, a high $\Gamma_{\text {air }}$ of $20.3 \%$ was achieved with the proposed quadruple structure. Furthermore, it was confirmed that the highest $\Gamma_{\text {air }}$ appears at $\mathrm{d}=400 \mathrm{~nm}$ and a tolerance range 
of $\pm 20 \%$, wherein the difference of $\Gamma_{\text {air }}$ is less than $2 \%$, which is helpful for facilitating etching. Meanwhile, a low scattering loss of $0.06 \mathrm{~dB} / \mathrm{cm}$ was estimated theoretically. The proposed quadruple structure high-mesa waveguide can realize future high-sensitivity devices for optical absorption spectroscopy.

\section{Acknowledgements}

The authors wish to thank Mr. Yasuaki Hashizume and Mr. Mikitaka Itoh of NTT Photonics Laboratories for their valuable comments and discussion. 